\title{
Hypereosinophilia Associated with Increased Serum Levels of Carcinoembryonic Antigen
}

\author{
Hiroka Iwata, Nobuo Tsuboi, Takeo Ishii, Yoichiro Hara, Ichiro Okido, Hajime Takahashi, \\ Tetsuya Kawamura and Tatsuo Hosoya
}

\begin{abstract}
We report a case of hypereosinophilia associated with increased serum levels of carcinoembryonic antigen (CEA). The patient developed fever, diarrhea, erythroderma and eosinophilia. Disorders known to be associated with eosinophilia were not detected. The typical malignant diseases related to a rise in CEA levels were not identified. The CEA value reached a maximum of $81.4 \mathrm{ng} / \mathrm{ml}$ a few weeks late for the peak of the eosinophilic count. Corticosteroid therapy was effective in improving clinical symptoms and the CEA values decreased in association with the improvement of those manifestations, suggesting a pathophysiological link between the disease activity of hypereosinophilia and the changes in CEA level.
\end{abstract}

Key words: eosinophilia, HES, CEA, corticosteroid

(Inter Med 47: 963-967, 2008)

(DOI: 10.2169/internalmedicine.47.0611)

\section{Introduction}

Hypereosinophilic syndrome (HES) is a rare disease characterized by persistent eosinophilia without any underlying causes, which tends to result in multiple systemic organ injuries. HES was first reported by Hardy and Anderson in 1968 (1). Chusid et al. proposed the inclusion criteria in 1975 (2). These criteria include the following three features: (i) persistent eosinophilia of $>1,500 / \mathrm{mm}^{3}$ for more than 6 months or death within 6 months with the signs and symptoms of hypereosinophilic disease; (ii) no evidence of any known causes of eosinophilia; and (iii) signs and symptoms of organ involvement, including hepatosplenomegaly, organic heart murmur, congestive heart failure, diffuse or focal central nervous system abnormalities, pulmonary fibrosis, fever, weight loss, and/or anemia.

Recent studies have established that some cases with hypereosinophilia may be explained by either to primitive involvement of myeloid cells essentially due to occurrence of an interstitial chromosomal deletion on $4 \mathrm{q} 12$ leading to the creation of the FIP1L1-PDGFRA fusion gene, or to increased interleukin- 5 production by a clonally expanded $\mathrm{T}$ cell population most frequently characterized by a CD3-CD-
4+ phenotype (3). FIP1L1-PDGFRA fusion gene is a constitutively activated tyrosine kinase that provides proliferative and survival signals and that can be inhibited by the small molecule kinase inhibitor imatinib. It is reported that FIP1L1-PDGFRA fusion gene positive patients show a dramatic response to imatinib therapy and most of them achieve complete hematological and molecular remission (4).

Several serum tumor markers have been evaluated in a variety of malignancies, and are mostly used to determine the evolution of tumors. However, it has recently been reported that tumor markers are also elevated in nonmalignant diseases, thus casting doubt on the specificity of the serum tumor markers. For example, it has been reported that CEA, a tumor marker for colon cancer, is elevated in patients with eosinophilia-related disorders (5-7). Although the pathophysiological role of elevated CEA in eosinophilia-related diseases is not clear, some reports indicate a close relationship between the disease activity and the changes in CEA levels (5-7). Here, we report a case of hypereosinophilia in which disease activity correlated with the serum levels of CEA. 

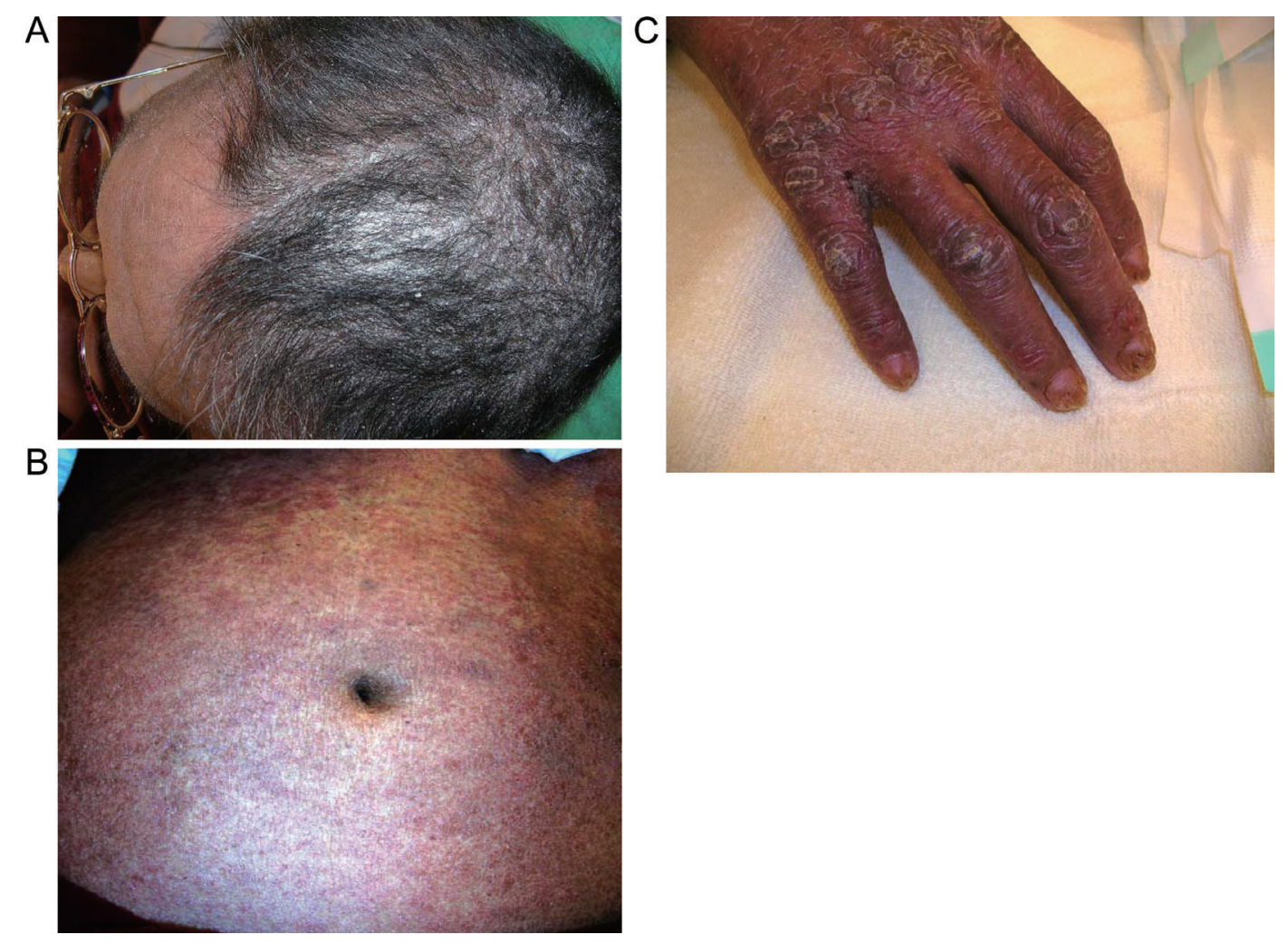

Figure 1. Cutis symptoms on admission. The patient shows epidermolysis of the scalp (A); and erythroderma with desquamation and erythema on the trunk and dorsum manus (B, C).

\section{Case Report}

The patient was a 44-year-old Japanese man with a medical history of severe non-insulin-dependent diabetes mellitus. He was under maintenance dialysis therapy because of chronic renal insufficiency due to diabetic nephropathy. At the beginning of June 2006, he experienced a high fever and frequent diarrhea. His doctor at the dialysis clinic prescribed Loxoprofen and Loperamide. Subsequently, systemic efflorescence developed. Because symptomatic improvement was not obtained, he was admitted to our hospital in mid-June. Since the drug lymphocyte stimulation test (DLST) with Loxoprofen was found to be positive three days later, he was initially diagnosed with drug eruption. He started oral administration of prednisolone at a dose of $30 \mathrm{mg}$ per day. High fever and cutis symptoms were immediately improved after ingestion of prednisolone. However, the clinical symptoms aggravated after tapered dosage of corticosteroid and he was hospitalized again in early August.

On admission, he was alert. His body temperature was $37.3^{\circ} \mathrm{C}$. His blood pressure was $142 / 92 \mathrm{mmHg}$. His palpebral conjunctiva was slightly anemic. He had no peripheral lymphadenopathy. Clinical examinations of the chest and abdomen revealed no abnormalities. He had erythroderma with desquamation and erythema over his whole body (Fig. 1).

Laboratory findings on admission were as follows: a white blood cell count (WBC) $5,200 / \mathrm{mm}^{3}$ with $12.3 \%$ eosinophils, hemoglobin (Hb) $9.1 \mathrm{~g} / \mathrm{dl}$, platelets (PLT) 23.9x
$10^{4} / \mu \mathrm{l}$. The erythrocyte sedimentation rate (ESR) was elevated at $104 \mathrm{~mm}$ per hour. Aspartate aminotransferase (AST) $15 \mathrm{IU} / \mathrm{L}$, alanine aminotransferase (ALT) $15 \mathrm{IU} / \mathrm{L}$, Lactate dehydrogenase (LDH) 439 IU/L, cholinesterase (ChE) $2121 \mathrm{IU} / \mathrm{ml}$, total bilirubin (T-Bil) $0.6 \mathrm{mg} / \mathrm{dl}$, alkaline phosphatase (ALP) 359 IU/L, $\gamma$-glutamyl transpeptidase $(\gamma$ GTP) $156 \mathrm{IU} / \mathrm{ml}$, total protein (TP) $8.2 \mathrm{~g} / \mathrm{dl}$, albumin (Alb) $3.8 \mathrm{~g} / \mathrm{dl}$ and C-reactive protein (CRP) $6.7 \mathrm{mg} / \mathrm{dl}$. The levels of serum immunoglobulins were as follows: IgG $1,862 \mathrm{mg} /$ dl, IgA $289 \mathrm{mg} / \mathrm{dl}$, IgM 46 mg/dl and IgE 24 IU/ml. Rheumatoid factor and various auto antibodies were all negative in the range that we examined. Fungal antigen tests were negative. Bacteria that cause diarrhea were not detected on stool culture examination. Helminth eggs were not detected in the stool. The serum CEA concentration on readmission was $31.8 \mathrm{ng} / \mathrm{ml}$ and showed aberrantly high levels. Other tumor markers did not show aberration in the range that we examined. Drug lymphocyte stimulation tests (DLST) against other medicines that he used regularly were all negative. These include lansoprazole, atrvastatin calcium, candesartan cilexetil, aspirin, allopurinol, nifedipine, sennoside, aiprazolam, furosemide, zolpidem, doxazosin mesilate, sevelamer hydrochloride and nitrazepam. The electrocardiogram and echocardiograph were normal. Computed tomography (CT) and echography showed no evidence of malignancies of the thyroid gland, chest, and digestive organs. Lymphadenopathy and hepatosplenomegaly were not evident. Endoscopic examination of the upper and lower gastrointestinal tract showed no evidence of malignancies. Figure 2 shows 
the findings of the skin biopsy taken from dorsum of the foot on August 24. Histology of the skin biopsy showed eosinophilic infiltration in the superficial layer of the corium. Ga-scintigraphy showed enhanced accumulation in both submandibular glands, but there was no laterality. Bone marrow aspirate did not show atypical cells or chromosomal aberration. Examination for human herpesvirus-6 (HHV-6) was not performed immediately after hospitalization. Several months later, polymerase chain reaction (PCR) for HHV-6 DNA was performed using peripheral blood and the result was negative.

Figure 3 shows the clinical course of the present case. The eosinophil count at the dialysis clinic in May 2006 was within the normal range. However, it increased remarkably in June 2006 when the patient suffered from pyrexia and diarrhea. Diagnosed as severe drug eruption due to Loxoprofen, oral prednisolone was started, at a dose of $30 \mathrm{mg}$ per day. Once corticosteroid administration was started, the

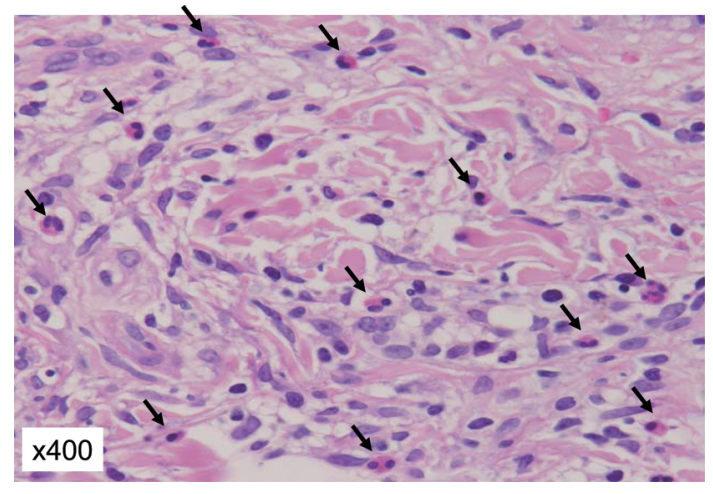

Figure 2. Skin biopsy taken from dorsum of the foot (Hematoxylin and Eosin staining, $\times \mathbf{4 0 0}$ original magnification). There is marked infiltration of eosinophils (arrows). eosinophil count decreased immediately. However, pyrexia, efflorescence, and eosinophilia recurred after the corticosteroid dosage was tapered. On readmission, the cause of pyrexia and eosinophilia was not clear despite a series of examinations. He was given oral prednisolone again at a dose of $20 \mathrm{mg}$ per day from September 9. At that time, in consideration of his severe diabetes mellitus and end stage renal failure, corticosteroid administration was started at a comparatively low dosage. Corticosteroid therapy resulted in reduction of the eosinophil count in peripheral blood and improvement of clinical symptoms including pyrexia and diarrhea. Notably, the serum CEA values, which showed an abnormally high level on admission, continued increasing afterward. It reached a maximum of $81.4 \mathrm{ng} / \mathrm{ml}$ a few weeks after the peak of the eosinophilic count. Interestingly, it showed a tendency to decrease after corticosteroid therapy was started and a decrease in the CEA levels was again found a few weeks after the decrease in eosinophils. By tapering corticosteroid, the eosinophil count showed a tendency to rise with pyrexia and it was followed again by a rise in serum CEA. Currently, about one year after the onset of disease, it is still difficult to reduce the dosage of corticosteroid because of the recurrence of the manifestations after tapering.

\section{Discussion}

Since our patient exhibited skin eruptions after the use of Loxoprofen and Loperamide, we postulated the possibility that our patient had drug-induced hypersensitivity syndrome (DIHS) which shows prolonged eruptions due to drugs. Recently, it has been recognized that DIHS, characterized by serious adverse systemic reactions and skin rash, is frequently associated with reactivation of human herpes virus 6

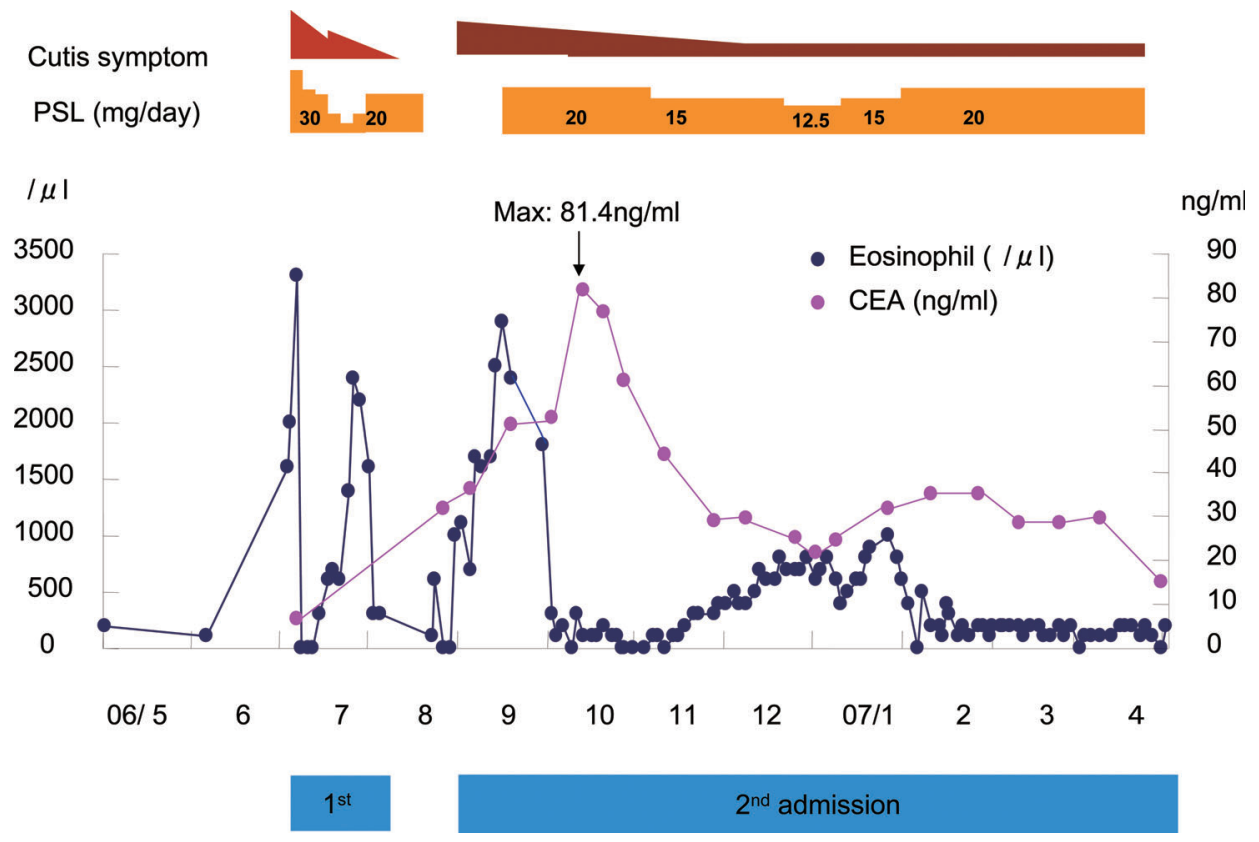

Figure 3. The clinical course. Note that corticosteroid therapy resulted in a decrease in serum CEA values in association with the improvement of eosinophilia. 
Table 1. Case Reports of Eosinophilia-related Diseases that Showed Abnormal CEA Elevations

\begin{tabular}{|c|c|c|c|c|c|}
\hline Author & Age & Sex & $\begin{array}{l}\text { Eosinophilia-related } \\
\text { diseases }\end{array}$ & $\begin{array}{l}\text { Maximum serum CEA } \\
\text { value }(\mathrm{ng} / \mathrm{ml})\end{array}$ & Reference \\
\hline Schottke et al. & 44 & $\mathrm{~F}$ & $\begin{array}{l}\text { Polypoid eosinophilic } \\
\text { pansinusitis }\end{array}$ & 85.0 & (5) \\
\hline Ashitani et al. & 70 & $\mathrm{~F}$ & Eosinophilic pneumonia & 17.1 & (6) \\
\hline Tsuburai et al. & 50 & $\mathrm{~F}$ & $\begin{array}{c}\text { Eosinophilic bronchitis and } \\
\text { bronchiolitis }\end{array}$ & 102.5 & (7) \\
\hline Present case & 44 & M & Hypereosinophilia & 81.4 & \\
\hline
\end{tabular}

(HHV6) (8). Reactivation of cytomegalovirus (CMV) or Epstein-Barr virus (EBV) has also been reported in patients with DIHS $(9,10)$. This syndrome typically develops within 2-6 weeks after taking drugs, and tends to persist for 2 weeks to several months after discontinuing the drugs. The most common reported drugs that cause DIHS are anticonvulsants, sulfasalazine, dapsone, minocycline, and mexiletine chloride (11-13). The main symptoms of DIHS are generalized exanthematous eruption, sometimes with small pustules, facial edema, high fever, systemic lymphadenopathy, leukocytosis, eosinophilia, atypical lymphocytosis and liver dysfunction $(11,14,15)$. In fact, the patient showed some of these manifestations. However, the present case was not typical of DIHS since the skin eruption developed immediately after taking drugs and neither Loxoprofen nor Loperamide was a major casual drug for DIHS. However, we could not perform the examination for HHV6 immediately after the onset of the disease. In addition, it is reported that there are interventions of comparatively rare drug and/or other viruses $(9,10)$. Thus, we can not exclude the possibility of DIHS as a cause of eruption and hypereosinophilia.

In the present case, the patient needed steroid therapy as soon as possible since his physical condition was worsening due to pyrexia and diarrhea. Therefore, this case does not satisfy the first criteria of HES: persistent eosinophilia of $>$ $1,500 / \mathrm{mm}^{3}$ for more than 6 months. However, even though 6 months have passed since the onset of disease, the eosinophil count has shown a tendency to rise and the skin eruptions have recurred after tapering the steroid dosage. Therefore, we considered our patient's condition to be essentially the same as that indicated in the first criteria of HES. However, we did not reach a decision for a cause of eosinophilia because we did not perform a search of the fused gene of FIP1L1/PDGFRA and examination for lymphocyte cell surface marker. Therefore this case does not reach a diagnosis with HES in the present conditions.

This case presented extremely high CEA levels. Although we considered the possibility that malignant diseases merged, a tumor was not found during the clinical course. We also considered about the possibilities of CEA rise due to other disease conditions such as diabetes and/or renal insufficiency seen in this case. However, it was difficult to explain the cause of such a drastic increase in CEA and its close correlation with the changes in eosinophil count only by diabetes and/or renal insufficiency. Some case reports have demonstrated abnormal CEA elevations in eosinophilia-related diseases (Table 1). Interestingly, most of these reports showed that elevated CEA values are decreased in association with the attenuation of the diseases, such as in the present case. These observations suggest a pathophysiological link between the changes in CEA levels and the activity of eosinophilia-related diseases. One of these reports on a respiratory system-related disorder with eosinophilia suggested that CEA secretion increased from bronchial epithelial cells and bronchi secreta (7). We examined the immunohistochemistry of the CEA using a skin biopsy sample to confirm the possibility that the skin of our patient was a source of serum CEA values. However, no significant levels of CEA were detected in either eosinophils or on other skin tissues (data not shown). A recent study showed that CEA levels on the eosinophil surface may increase when eosinophils are activated by cytokines (16). Although the cause of elevated CEA levels has not been determined at present, a mechanism such as eosinophil activation may underlie the abnormal CEA elevation observed in our case. On the other hand, it is possible that other unknown factors related to eosinophilia indirectly regulated CEA elevation, which is supported by a report on HES showing normal CEA values despite extremely high peripheral blood eosinophil counts (17).

\section{References}


2. Chusid MJ, Dale DC, West BC, Wolff SM. The hypereosinophilic syndrome. Analysis of fourteen cases with review of the literature. Medicine 54: 1-27, 1975.

3. Gotlib J, Cools J, Malone JM 3rd, Schrier SL, Gilliland DG, Coutre SE. The FIP1L1-PDGFR $\alpha$ fusion tyrosine kinase in hypereosinophilic syndrome and chronic eosinophilic leukemia: implications for diagnosis, classification, and management. Blood 103 (8): 2879-2891, 2004.

4. Cools J, DeAngelo DJ, Gotlib J, et al. A tyrosine kinase created by fusion of the PDGFRA and FIP1L1 genes as a therapeutic target of imatinib in idiopathic hypereosinophilic syndrome. N Engl J Med 348 (13): 1201-1214, 2003.

5. Shottke H, Padberg BC. Differential diagnosis of destructive processes of the skull base. Aggressive polypoid eosinophilic pansinusitis with expression of carcino-embryonic antigen (CEA). HNO 38 (5): 170-173, 1990 (in German, Abstract in English).

6. Ashitani J, Sakamoto A, Maki H, et al. A case of eosinophilic pneumonia with elevated levels of carcino-embryonic antigen (CEA). Nihon Kyobu Shikkan Gakkai Zasshi 32 (12): 1194-1198, 1994 (in Japanese, Abstract in English).

7. Tsuburai T, Kawabata Y, Tsurikisawa N, et al. Case of eosinophilic bronchitis and bronchiolitis associated with increased level of serum CEA in asthmatics. Nihon Kokyuki Gakkai Zasshi 44 (10): 742-748, 2006 (in Japanese, Abstract in English).

8. Descamps V, Bouscarat F, Laglenne S, et al. Human herpesvirus 6 infection associated with anticonvulsant hypersensitivity syndrome and reactive haemophagocytic syndrome. Br J Dermatol 137: 605608, 1997.

9. Aihara M, Sugita Y, Takahashi S, et al. Anticonvulsant hypersensitivity syndrome associated with reactivation of cytomegalovirus.
Br J Dermatol 144: 1231-1234, 2001.

10. Descamps V, Mahe E, Houhou N, et al. Drug-induced hypersensitivity syndrome associated with Epstein-Barr virus infection. Br J Dermatol 148: 1032-1034, 2003.

11. Shiohara T, Inaoka M, Kano Y. Drug-induced hypersensitivity syndrome (DIHS): A reaction induced by a complex interplay amoung herpesviruses and antiviral and antidrug immune responses. Allergology Int 55 (1): 1-8, 2006.

12. Callot V, Roujeau JC, Bagot M, et al. Drug-induced pseudolymphoma and hypersensitivity syndrome. Two different clinical entities. Arch Dematol 132 (11): 1315-1321, 1996.

13. Carroll MC, Yueng-Yue KA, Esterly NB, Drolet BA. Druginduced hypersensitivity syndrome in pediatric patients. Pediatrics 108 (2): 485-492, 2001.

14. Mitani N, Aihara M, Yamakawa Y, et al. 2005 Drug-Induced hypersensitivity syndrome due to cyanamide associated with multiple reactivation of human herpesviruses. J Med Virol 75 (3): 430-434, 2005.

15. Yagami A, Yoshikawa T, Asano Y, Koie S, Shiohara T, Matsunaga K. Drug-induced hypersensitivity syndrome due to mexiletine hydrochloride associated with reactivation of human herpesvirus 7 . Dermatology 213 (4): 341-344, 2006.

16. Mawhorter SD, Stephany DA, Ottesen EA, Nutman TB. Identification of surface molecules associated with physiologic activation of eosinophils. Application of whole-blood flow cytometry to eosinophils. J Immunol 156 (12): 4851-4858, 1996.

17. Inoue A, Michitaka K, Shigematsu S, et al. Budd-Chiari syndrome associated with hypereosinophilic syndrome; A case report. Intern Med 46 (14): 1095-1100, 2007.

(C) 2008 The Japanese Society of Internal Medicine http://www.naika.or.jp/imindex.html 